\title{
Dismorfia muscular: definición y generalidades sobre prevención y tratamiento.
}

Muscle dysmorphia: definition and general information about prevention and treatment.

\author{
Diana B. Torres-Mota ${ }^{a}$, Itzehl Pontaza-Ortiz ${ }^{b}$, María Fernanda Alvarez-Espinoza ${ }^{c}$, Teresita \\ D.J. Saucedo-Molina ${ }^{d}$
}

\begin{abstract}
:
Muscle dysmorphia (DM) is understood as a disorder characterized by excessive concern about body image in which the person has a distortion specifically in relation to their muscle size; there is a higher prevalence in men between 18 to 30 years old. According to DSM-5, DM represents a specific type of body dysmorpic disorder; this is influenced by different psychological, social and biological risky factors. In this pathology, the frequent consumption of ergogenic substances with the intention to increased muscle mass is common, having a main impact on the health of those who consume them. The treatment of this disorder involves a multidisciplinary team of highly trained professionals in a way that they can correctly recognize the diagnostic characteristics to provide a correct diagnosis and adequate treatment. This treatment includes psychoeducation based on cognitive dissonance, nutritional orientation and in some cases the help of drugs such as antidepressants or antipsychotics. The present work describes the historical antecedents of muscle dysmorphia, the criteria for its diagnosis, as well as generalities about its prevalence, prevention and treatment.
\end{abstract}

Keywords:

Muscle dysmorphia, risky factors, ergogenic substances, drive for muscularity

\section{Resumen:}

La dismorfia muscular (DM) se entiende como un trastorno que se caracteriza por una preocupación excesiva sobre la imagen corporal en la cual la persona presenta una distorsión concretamente en relación de su tamaño muscular; hay una mayor prevalencia en hombres de 18 a 30 años de edad. De acuerdo al DSM-5, la DM representa un tipo específico del trastorno dismórfico corporal; esta se encuentra influenciada por diferentes factores de riesgo tales como psicológicos, sociales y biológicos. En esta patología es común el consumo de sustancias ergogénicas en busca del aumento de masa muscular, teniendo un gran impacto sobre la salud de quien los consume. El tratamiento de este trastorno implica la atención de un equipo multidisciplinario de profesionales altamente capacitados para que puedan reconocer correctamente las características propias y así proporcionar un diagnóstico correcto y el tratamiento adecuado. En dicho tratamiento se incluye la psicoeducación basada en la disonancia cognitiva, orientación nutricional y en algunos casos la ayuda de fármacos como antidepresivos o antipsicóticos. En el presente trabajo se describen los antecedentes históricos de la dismorfia muscular, los criterios para su diagnóstico, así como también generalidades sobre su prevalencia, prevención y tratamiento.

\section{Palabras Clave:}

Dismorfia muscular, factores de riesgo, sustancias ergogénicas, obsesión por la musculatura

\section{Introducción}

La Dismorfia Muscular (DM), conocida coloquialmente como "vigorexia", es un trastorno caracterizado por una preocupación excesiva con la idea de no tener uncuerpo suficientemente magro o musculoso y que puede conllevar a renunciar a las actividades sociales y laborales por la necesidad de ejercitarse ${ }^{1}$.

\footnotetext{
${ }^{a}$ Universidad Autónoma del Estado de Hidalgo, https://orcid.org/0000-0002-5539-0291, Email: dianabtmo@ hotmail.com

${ }^{\mathrm{b}}$ Universidad Autónoma del Estado de Hidalgo, https://orcid.org/0000-0003.3134-225X, Email: itzehlpontaza@gmail.com

c Universidad Autónoma del Estado de Hidalgo, https://orcid.org/0000-0001-8845-6894, Email: mafer_al3007@hotmail.com

d Autor de correspondencia, Universidad Autónoma del Estado de Hidalgo, https://orcid.org/0000-0001-8597-5349, Email: 
La persona que presenta dicho trastorno realiza ejercicios de fuerza de una manera compulsiva, con el objetivo de aumentar su tamaño muscular, pudiendo desarrollar una dependencia ${ }^{2}$. Esta dependencia 0 adicción al entrenamiento de fuerza genera en el individuo la necesidad de constatar su hipertrofia y definición muscular continuamente.

En la década de los años 90 del siglo XX, Pope y Katz ${ }^{3}$ investigadores de la Universidad de Harvard, estudiaban los efectos secundarios del uso de esteroides anabolizantes por usuarios de gimnasio con el objetivo de conseguir un mayor desarrollo muscular, descubriendo en algunos de ellos que se podía esconder un nuevo trastorno psicológico. Dicha patología se caracterizaba por un obsesivo deseo de desarrollo muscular, pero con una permanente insatisfacción del tamaño del cuerpo alcanzado debido a una distorsión de la imagen corporal, de tal modo que alteraban significativamente su estilo de vida con la intención de conseguir mayor masa muscular al pasar más tiempo ejercitándose en el gimnasio junto con el uso exagerado de ayudas ergogénicas, tales como las sustancias anabolizantes.

Debido a que tenía un parecido a la Anorexia Nerviosa, pero con características opuestas como el querer ser más grandes y comer más, se le denominó Anorexia Inversa ${ }^{3}$.

Dado que la DM no figuraba dentro de las clasificaciones del DSM-4-TR (American Psychiatric Association, APA, 1994), existió una controversia respecto a su clasificación, de tal modo que se creyó que podría ubicarse dentro de los Trastornos de la Conducta Alimentaria (TCA ${ }^{1}$. Igualmente fue considerado como un Trastorno de Ansiedad, concretamente como un Trastorno Obsesivo Compulsivo debido al entrenamiento riguroso al que se someten los pacientes, y a las dietas estrictas, específicamente de tipo hiperproteicas e hipercalóricas; Martínez, Cortés, Rizo y Gil (2015) ${ }^{4}$ determinaron que los valores de proteína y el consumo de colesterol sobrepasan los límites propuestos para personas que desean desarrollar masa muscular. Finalmente, podría ubicarse dentro de los Trastornos Somatomorfos, concretamente del Trastorno Dismórfico Corporal debido a la excesiva preocupación por la imagen corporal.

\section{Criterios de Diagnóstico}

En la más reciente publicación del DSM-5 (American Psychiatric Association, APA, 2013) $)^{5}$ aparece la DM dentro del capítulo de los Trastornos Obsesivos Compulsivos y otros trastornos relacionados, donde los criterios de diagnóstico se encuentran en la Tabla 1.
Tabla 1. Criterios de diagnóstico de dismorfia muscular de acuerdo con DSM-5.

Criterios de diagnóstico de dismorfia muscular $(\mathrm{A}+\mathrm{B})$

A. Cumple criterios de Trastorno Dismórfico Corporal;

B. Preocupación excesiva con el cuerpo, considerando que sus formas corporales son muy pequeñas o no lo suficientemente musculosas.

\section{Trastorno Dismórfico Corporal}

1. Preocupación con uno o más defectos 0 alteraciones físicas percibidas por el paciente, pero no observadas por otros individuos.

2. En algún momento en el curso de la enfermedad, el individuo realiza comportamientos 0 actos mentales repetitivos en respuesta a la preocupación con la apariencia física.

3. La preocupación con el cuerpo causa sufrimiento clínicamente significativo o perjuicio en el funcionamiento social, ocupacional o en otras áreas importantes de funcionamiento.

4. La preocupación con la apariencia no satisface criterios de diagnóstico para una perturbación del comportamiento alimentario.

Además de los criterios señalados, también se menciona que los individuos que presentan dicho trastorno, pueden estar preocupados por otras áreas de su cuerpo, como la piel y el cabello, además de realizar dietas restrictivas, ejercicio excesivo como levantamiento de pesas, así como el consumo de esteroides anabólicos-androgénicos u otras sustancias potencialmente peligrosas, pudiendo provocar daño corporal, daño mental e incluso la muerte ${ }^{1}$.

\section{Factores de riesgo}

Como se mencionó anteriormente, la DM incluye características obsesivas compulsivas las cuales son muy frecuentes en los $\mathrm{TCA}^{6}$.

Dentro de los principales factores de riesgo se encuentra la excesiva atención que le prestan a su dieta, pues se adhieren a planes dietéticos muy rígidos, siendo muy altos en proteínas (hasta $5 \mathrm{~g}$ de proteína/kg) ${ }^{6}$, y bajos en grasas, de manera que les es complicado comer fuera de casa pues les resulta difícil calcular cada alimento que van a consumir; además comen continuamente, incluso aunque no sientan hambre. 
Como factores psicológicos existe la insatisfacción con la imagen corporal, la baja autoestima y el perfeccionismo ${ }^{7}$. La imagen corporal es aquella que crea nuestra mente sobre nuestro propio cuerpo ${ }^{8}$, esta se encuentra conformada por 4 componentes: el componente perceptual (percepción del cuerpo en su totalidad o de alguna de sus partes), el componente cognitivo (pensamientos y creencias sobre el cuerpo), el componente afectivo (sentimientos y grado de satisfacción) y el componente conductual (acciones o comportamientos que se dan a partir de la percepción corporal) ${ }^{9}$.

La sociedad actual transmite modelos de perfección corporal mediante la presión ejercida por los medios de comunicación, la publicidad y el fenómeno de la imitación, que promueven la adopción de actitudes (emociones, cogniciones y conductas) que son generadas hacia individuos que representan figuras de respeto 0 admiración y pueden convertirse en un factor de riesgo para el desarrollo de alteraciones de la imagen corporal ${ }^{10}$.

La publicidad, en sus distintas formas y presentaciones, se ha integrado en nuestra sociedad de un modo indisoluble y cotidiano, e igualmente se ha convertido en un elemento imprescindible ${ }^{11}$. Hoy en día juega un papel importante en cuanto al desarrollo económico y social de los países en general. Esta forma de marketing, sobre todo de productos estéticos, presenta al cuerpo como un objeto que se puede modelar fácilmente y conseguir que se asemeje al ideal estereotipado. Se presentan diferentes productos y servicios que predisponen a ese cambio anhelado y soñado por muchos hombres y mujeres, pero la realidad es distinta. La idea de que nuestro cuerpo es un objeto modelable y fácilmente modificable es la consecuencia de una sociedad consumista y hedonista que ha creado un mercado empresarial movilizado para vender ilusión ${ }^{12}$.

El aspecto físico de determinados modelos publicitarios constituye un código no verbal del mensaje comercial que participa activamente en el proceso de significancia, y que atribuye al producto anunciado una serie de valores que transmutan el sentido final de la comunicación. Los cuerpos magros y musculosos de los modelos masculinos que imperan en determinados tipos de anuncios transmiten ideas estéticas de belleza que van ligadas de forma connotada, valores socialmente deseables tales como el éxito, poder, estatus, entre otras. Este aspecto físico masculino, promulgado masivamente por la publicidad y los medios de comunicación, es usualmente buscado por los hombres, ya sea de manera consciente 0 inconsciente ${ }^{11}$. Estos modelos publicitarios "dominantes" ejercen gran presión para realizar ejercicio físico excesivo con la finalidad de alcanzarlos.

Existe también la cultura de la delgadez que habla de la presión que generan las exigencias del ideal de belleza, dentro de esto podemos encontrar a la delgadez y a la "figura perfecta" que se presentan como valores positivos asociados con condiciones para tener éxito, ir a la moda y ser reconocidos socialmente; contrariamente, los individuos tienden a asociar el sobrepeso con fracaso, descuido, desorden y poco control. La delgadez también ha sido asociada a la seguridad y autocontrol, en este sentido el mantener una figura perfecta es visto como un indicador de incremento de autoestima ${ }^{12}$. Este efecto produce una distorsión de la imagen corporal ya que uno de los determinantes de la cultura de la delgadez son las tallas ideales, el cual a través de diferentes medios publicitarios tales como la televisión y las revistas transmiten con diferentes mensajes que incitan directa o indirectamente a perder peso en las mujeres y a incrementar la masa muscular en los varones.

Por otro lado, existe la posibilidad de que la violencia juegue un papel importante para el desarrollo de la DM, como el haber sido víctima de experiencias traumáticas, ya sea el abuso físico o sexual, el acoso escolar (bullying) o la intimidación durante la infancia ${ }^{13}$, sin embargo, no se presenta en todos estos casos.

De acuerdo a Muñoz y Gómez (2003) ${ }^{14}$, se ha propuesto una tendencia genética a la DM, considerada como una adicción. De acuerdo a Alonso $(1996)^{15}$, el bajo rendimiento del sistema serotoninérgico puede aparecer junto con la DM, ocasionando el desequilibrio de otros neurotransmisores como el GABA (encargado de la inhibición específica de comportamiento y la respuesta del cuerpo frente al estrés), o la dopamina (cuyos niveles aumentan significativamente durante la práctica excesiva de deporte). Martell $(2006)^{16}$ refiere otra explicación biológica que implica al sistema nervioso simpático y la dependencia de niveles superiores de catecolaminas que acompañan a la práctica de ejercicio. También sugieren que dicho trastorno puede ser el resultado de un déficit de opioides endógenos, estimulados por el ejercicio.

La práctica compulsiva de deporte proporciona placer, aumentando la autoestima y mejorando las relaciones sociales, resultando en un reforzamiento positivo, pues se reduce la ansiedad y el estrés. Los sentimientos negativos que se presentan en estas personas cuando no realizan deporte son similares a los de la abstinencia, pues la práctica de ejercicio es un proceso potencialmente adictivo. Yates $(1991)^{17}$ menciona que los sujetos adictos al ejercicio muestran rasgos de tipo egosintónico, ya que 
obtienen placer con su comportamiento, pero los sujetos obsesivo-compulsivos son egodistónicos, ya que no obtienen placer, sino que, al presentarse un malestar previo, utilizan la actividad física como reductora de dicho malestar. En otras palabras, las personas que padecen DM no desarrollan este reforzamiento positivo, pues no disfrutan de realizar ejercicio, sino que evitan el malestar que les produce el no realizarlo.

\section{Obsesión por la Musculatura}

Uno de los factores de riesgo más asociados a la aparición de la DM es la denominada obsesión por la musculatura (OM); dicho término fue acuñado por McCreary y colaboradores, y se caracteriza por la preocupación excesiva por la musculatura con respecto al tamaño corporal y los intentos por ganar peso y la pérdida de grasa $^{18}$.

La Escala de Obsesión por la Musculatura (DMS, por sus siglas en inglés), es un cuestionario que consta de 15 ítems que permite evaluar la medida en que la gente desea tener un cuerpo más musculoso. Estos ítems corresponden a la combinación de comportamientos y actitudes, y se califica en una escala que va desde siempre hasta nunca; aquellas puntuaciones más altas corresponden a una mayor obsesión por la musculatura. La versión mexicana cuenta con tres subescalas: la primera es la obsesión por incrementar la musculatura (coeficiente de alpha 0.9), la segunda es el uso de suplementos alimenticios (coeficiente de alpha 0.7), la tercera es la obsesión por el entrenamiento (coeficiente de alpha 0.5). Para esta escala se ha propuesto un punto de corte igual o mayor a $52^{19}$.

En estudios realizados en Hidalgo, Arellano-Pérez, Vázquez-Cervantes, Fernández-Cortés y Saucedo-Molina $(2019)^{18}$, lograron identificar que un $14,2 \%$ de una muestra de 267 hombres adolescentes de entre 15-19 años de edad presentaban riesgo de desarrollar OM, con un puntaje de corte de $\geq 52$ para esta escala; además determinaron la relación de indicadores antropométricos (IMC, porcentaje de grasa corporal y masa muscular) y AF con el riesgo de desarrollar conductas alimentarias de riesgo (CAR) y OM, encontrando que no hubo diferencia estadísticamente significativa de desarrollar OM con respecto al IMC. Además, se pudo determinar que los adolescentes con IMC normal mostraron un porcentaje más alto de riesgo de desarrollar $\mathrm{OM}$, mientras que aquellos ubicados en obesidad presentaban riesgo más alto para el desarrollo de CAR, pues estas personas al buscar la reducción de peso utilizan estrategias que no siempre son las adecuadas ni saludables para lograr ese objetivo. Respecto a la AF, el porcentaje más alto de personas en riesgo de desarrollar $\mathrm{OM}$ se identificó en aquellas que realizan AF moderada.

\section{Sustancias ergogénicas}

El ejercicio físico es una variedad de actividad física planeada, estructurada, repetitiva y realizada que tiene por objetivo el mantenimiento de uno o más componentes de la aptitud física ${ }^{20}$. La práctica regular de este, proporciona una serie de beneficios para la salud tanto física como psicosocial del individuo ${ }^{21}$. Sin embargo, las personas con DM adoptan conductas extremas con la finalidad de lograr cambios corporales, dentro de las cuales destaca el ejercicio físico como una conducta adictiva y como un entorno potencial de consumo de determinadas sustancias ergogénicas ${ }^{22}$.

Las sustancias ergogénicas son aquellas que mejoran el rendimiento de un organismo y son consideradas como cualquier método nutricional, físico, mecánico, psicológico o farmacológico utilizadas con el fin de aumentar la capacidad para realizar un trabajo físico y mejorar su desempeño $0^{23}$. Estas suelen clasificarse en: nutricionales (como los suplementos), farmacológicas (como las anfetaminas) $u$ hormonales (como los esteroides anabólicos androgénicos [EAA]) ${ }^{24}$.

Nutricionales (suplementos): Se caracterizan por ser un producto que puede ser usado para tratar las exigencias fisiológicas o nutricionales que se plantean en el deporte. De igual manera, pueden proporcionar un medio conveniente o práctico de aportar los requerimientos nutricionales especiales para el ejercicio, o pueden ser utilizados para prevenir o revertir las deficiencias nutricionales que ocurren comúnmente entre los atletas ${ }^{25}$. Sin embargo, el uso excesivo de estas sustancias puede desencadenar diferentes eventos tales como el daño renal, disfunción hepática aguda, lesión aguda colestática y desórdenes cardiovasculares (presión sanguínea elevada y ritmo cardiaco e infarto agudo de miocardio) así como también efectos adversos autopercibidos o no por el sujeto tales como dolor de cabeza y taquicardia ${ }^{26}$.

En un estudio realizado por la Universidad Autónoma de Nuevo León se menciona que el consumo de suplementos alimenticios en la población de México va incrementando. En este mismo se menciona que los suplementos más consumidos fueron las vitaminas, minerales, productos herbolarios, bebidas energetizantes y té verde. El principal propósito del consumo de suplementos encontrado fue para lograr un aumento de energía, aumento de masa muscular y para bajar de peso. El 16\% de esta población 
menciona haber tenido efectos adversos como: náuseas, mareos, debilidad y vómitos ${ }^{27}$.

Anfetamina: Estimulante del sistema nervioso central ${ }^{28}$. Entre los beneficios ergogénicos, se ha descubierto que tienen efectos psicológicos puesto que aumentan la concentración y la alerta mental. Sin embargo, también se han reportado como efectos adversos niveles altos de ansiedad. Su efecto estimulante es disminuir la fatiga. Se ha demostrado que las personas que toman anfetaminas experimentan: reducción de la sensación de fatiga, aumento en la frecuencia cardiaca e incremento en la tensión arterial. La utilización de estas es muy peligrosa ya que debido al incremento de frecuencia cardiaca y la tensión arterial se pueden provocar fallas en el corazón y llegar hasta la muerte ${ }^{29}$.

Esteroides anabólicos androgénicos: son derivados sintéticos de la testosterona, que estimulan la producción celular de proteínas, provocando un aumento en el tamaño muscular, generado por un aumento de la fibra y un desarrollo de las capacidades físicas condicionales (fuerza, velocidad, resistencia, flexibilidad) ${ }^{30}$.

El uso de estas sustancias en general, es indiscriminado, sin orientación específica por algún profesional altamente capacitado y ético. Entre los efectos adversos pueden señalarse aquellos relativos a los sistemas: hepático, cardiovascular, endocrino y reproductor. Dentro de las principales afecciones se encuentran: retención sódica, acné, ginecomastia, hipertensión, hipercolesterolemia, palpitaciones, agrandamiento del corazón, cáncer, hemorragias, dolores de cabeza, dolores de estómago, ictericia, desgarros musculares, agrandamiento de la próstata, calvicie prematura, supresión del sistema inmunológico, insomnio, atrofia testicular y posibles disfunciones sexuales; en cuanto a los efectos psicológicos más destacados se indican la euforia e irritabilidad, hiperactividad y estados depresivos ${ }^{26}$.

\section{Prevalencia}

No hay datos definitivos sobre la prevalencia de DM, a pesar de ello, se pueden citar datos al respecto:

- Pope, Phillips y Olivardia $(2000)^{31}$, sugirieron alrededor de 10,000 personas en el mundo que podrían encontrarse en esta situación, siendo más común en hombres.

- De acuerdo a Pope $(2000)^{31}$, este problema se presenta en diversas culturas.

- La DM se observa en ambos sexos, sin embargo, predomina en el sexo masculino ${ }^{7}$.
- La DM se presenta con mayor prevalencia entre los 18-35 años ${ }^{4}$.

- Pope $(2002)^{32}$, describió una prevalencia de DM de $10 \%$ en levantadores de pesas y usuarios de gimnasios y hasta el $84 \%$ entre culturistas que participan en competencias, siendo estos grupos la población de mayor riesgo para el desarrollo la misma.

- Olivardia $(2001)^{33}$, estimó que el número de norteamericanos con problemas de DM era de 90,000; siendo el mismo número de casos encontrado en México de acuerdo a Baile $(2005)^{34}$, mientras que en España se estimó "clínicamente" unos 700,000 casos según García-Gómez y Toledo-Romero (2005) ${ }^{35}$.

- La Unión Europea estimó un 6\% en los usuarios habituales de gimnasios con dicho trastorno (Méndez, 2002) ${ }^{36}$.

\section{Prevención}

La prevención es una herramienta importante y muy potente si está bien dirigida para evitar, disminuir o detener la aparición y el desarrollo de diversas enfermedades, tales como la DM. El realizar campañas de prevención, que incluyan actividades en torno a la imagen corporal, habilidades sociales, análisis y crítica de los ideales de belleza, aumento de autoestima, e información de aspectos relacionados con la nutrición y los hábitos alimentarios, puede evitar que multitud de personas sufran un trastorno de este tipo ${ }^{37}$.

En los programas de prevención orientados hacia la DM, resultaría útil analizar y criticar el modelo estético imperante, profundizar en el conocimiento sobre el desarrollo corporal masculino, desde la fisiología, y someter a juicio a los diferentes productos y dietas a los que hacen publicidad con el objetivo de desarrollar la masa muscular, así como el consumo de EAA con el mismo objetivo ${ }^{37}$.

A nivel social, se requiere un cambio de valores en cuanto a la cultura de la delgadez, evitando el modelo del hombre musculoso como ejemplo estético en campañas de publicidad, medios de comunicación, entre otros. Así como la concientización de la sociedad sobre la DM y los peligros derivados de la misma a partir de campañas dirigidas a la población desde organismos gubernamentales.

La educación y la formación de los profesionales de la salud de atención primaria en esta patología es la base de la prevención frente a la dismorfia muscular. Es necesario realizar una concientización hacia la población 
y hacia los profesionales de la salud acerca de este trastorno, informando sobre qué es, sus principales signos y síntomas para poder realizar una detección temprana, así como informar acerca de los riesgos del consumo indiscriminado de dietas o suplementos hiperproteicos y de EAA para poder llevar a cabo una amplia concientización en escuelas, gimnasios, y centros deportivos acerca de los riesgos de estas prácticas.

Resulta indispensable la formación de profesionales sanitarios de atención primaria en esta patología, así como educación sanitaria en instituciones donde se incluyan temas como el desarrollo corporal normal y patológico, el modelo estético masculino actual, dietas y productos para mejorar el desarrollo muscular, el uso de esteroides anabolizantes, así como qué es la DM, qué problemas puede producir y cómo se puede evitar.

A nivel individual, para que una consultoría nutricional resulte provechosa, es fundamental identificar conductas alimentarias de riesgo, investigar acerca de la percepción de la imagen corporal, junto con la realización de ejercicio, tiempos y duración, así como hábitos alimentarios, todo esto con el fin de otorgar orientación acerca de la enfermedad y si hay sospecha de esta poder realizar un diagnóstico y tratamiento $\operatorname{precoz}^{38}$.

En estudiantes universitarios, se implementó un programa de prevención universal: The Body Project: More than Muscles, con un total de 112 participantes varones. El objetivo de este estudio fue determinar la aceptabilidad y la eficacia de una intervención basada en disonancia cognitiva (DC) para reducir los factores de riesgo de trastornos de la conducta alimentaria y dismorfia muscular en hombres con insatisfacción corporal. Para la intervención, se formaron 2 grupos de manera aleatoria, en el grupo 1 recibieron la intervención grupal basada en DC y el grupo 2 que estaba en una condición de lista de espera. Se realizaron 2 sesiones grupales con una duración de 2 horas y que estuvieron separadas por una semana. En la sesión 1, los participantes: definieron el tipo de cuerpo masculino "ideal cultural" y discutieron el origen y la perpetuación de la visualización de imágenes (como los superhéroes de televisión ( películas) y que han cambiado con el tiempo, realizaron una lluvia de ideas sobre los costos de perseguir el "ideal cultural", participaron en desafíos verbales para contrarrestar el mensaje ideal mesomórfico, y completaron las asignaciones de tarea (un correo electrónico a un chico de la escuela secundaria, dos desafíos de comportamiento y una asignación de exposición al espejo). En la sesión 2, los participantes: revisaron la tarea, completaron juegos de roles para contrarrestar / desalentar la búsqueda del ideal mesomórfico, enumeraron formas de resistir la presión para perseguir este "ideal cultural" individualmente, como grupo, y dentro de la comunidad en general (denominado "activismo corporal"). Los participantes asignados al azar a la condición DC informaron reducciones significativas a las 4 semanas de seguimiento en los factores de riesgo y síntomas de trastornos de la conducta y en factores de riesgo y síntomas de dismorfia muscular en relación con la lista de espera. Los hallazgos de esta investigación tienen el potencial de ayudar a reducir el estigma en torno a las preocupaciones sobre la imagen corporal de los hombres y ayudar a mejorar la atención de un grupo que se ha pasado por alto durante demasiado tiempo ${ }^{39}$.

En el Estado de Hidalgo, se llevó a cabo un estudio de campo no experimental de medidas repetidas con un solo grupo (pre-test-, post-test y seguimiento de 6 meses), en donde participaron 214 mujeres y 154 hombres adolescentes. El objetivo fue evaluar los efectos de un programa de prevención universal de conductas alimentarias no saludables y sedentarismo. El programa, basado en la disonancia cognitiva junto con estrategias psicoeducativas interactivas, y conocido como Prevención de Conductas Alimentarias No Saludables y Sedentarismo (PECANSS) consiste en 5 sesiones impartidas en días consecutivos; las primeras 4 sesiones, con una duración de una hora, eran seguidas por otra hora de actividad física lúdica; la última sesión con una duración de 90 minutos, consistía en 3 talleres paralelos. En la primera sesión se discutió la cultura de la delgadez, con el objetivo de crear pensamientos para lograr un cambio de actitudes sin ser conscientes de ello. La sesión 2 abordó mitos sobre dietas y suplementos, además de que se discutió sobre los trastornos alimentarios más comunes y la DM. En la sesión 3 y 4 , se trataron temas sobre alimentación, nutrición y conductas alimentarias saludables. Finalmente, en la sesión 5 llamada multiactividades, se trabajó en equipos y se realizaron carteles en contra de la cultura de la delgadez, se diseñaron menús saludables y se llevó a cabo un rally deportivo.

Después de 6 meses, los análisis de varianza de medidas repetidas revelaron que hubo una reducción significativa en las puntuaciones medias de las conductas alimentarias de riesgo en las adolescentes. En los hombres adolescentes, las puntuaciones medias de conductas alimentarias de riesgo y de obsesión por la musculatura disminuyeron, pero no significativamente. La frecuencia y duración de la actividad física mostraron un aumento significativo en la muestra total a lo largo del tiempo. En general, la implementación del programa tuvo efectos positivos en los jóvenes y se encontraron diferencias importantes entre los sexos ${ }^{40}$. 


\section{Tratamiento}

Resulta necesario un equipo multidisciplinario, con el establecimiento de una alianza terapéutica sólida, además de psicoeducación de tal modo que permita reconocer las conductas de riesgo para mitigar la ansiedad ${ }^{7}$.

Psicoterapia: Se recomienda la psicoterapia conductivoconductual, con el objetivo de modificar los patrones de pensamiento obsesivo y fomentar habilidades de confrontamiento a problemas. Puede trabajarse de manera individual $\mathrm{y} / \mathrm{o}$ grupal, de entre 6-12 sesiones con una duración de 60-90 minutos; se puede reforzar con materiales de autoayuda. Esta terapia ha demostrado una eficacia en la reducción de la depresión, un aumento de la introspección y mejoría en la percepción de la imagen corporal ${ }^{41}$.

Psicofarmacología: El tratamiento de elección son antidepresivos como los inhibidores de la recaptura de serotonina en dosis altas, y cuyos efectos secundarios son bien tolerados en la mayoría de las veces. Dentro de estos se pueden encontrar a la fluoxetina, paroxetina, fluvoxamina y la sertralina ${ }^{42}$. Como fármaco de segunda línea se indica un tricíclico como la clopramina, aunque su tolerancia en cuanto a los efectos secundarios es menor, pues son más fuertes ${ }^{43}$.

La quetiapina también se usa como un fármaco para tratar episodios de manía, ideas delirantes y depresión en pacientes que presentan conjuntamente trastorno bipolar. Se ha reportado que el uso de antipsicóticos podría ser útil en pacientes que asocian ideas delirantes 0 un componente de agitación; sin embargo, aún no está bien establecido ${ }^{7}$.

La duración del tratamiento se recomienda que sea de 3 a 4 años, y en situaciones más graves el tiempo es indefinido. En caso de que se presente una recaída, se sugiere reiniciar con el tratamiento previo ${ }^{44}$.

Nutricional: La finalidad del tratamiento nutricional es el de conseguir un peso que no ponga en riesgo la salud del paciente, proporcionar educación nutricional para instaurar un comportamiento alimentario saludable y así normalizar aspectos psicológicos que mejoren las actitudes hacia la alimentación y la aceptación del propio cuerpo $^{45}$.

Durante el tratamiento nutricio es importante ayudar al paciente a identificar todos los beneficios que va logrando conforme se va acercando a una alimentación saludable tales como, disminución de riesgo de enfermedades no trasmisibles, sentirse con más energía, mejora del sistema inmunológico, mejora en sus funciones cognitivas.
Se recomienda una dieta cuyo contenido energético no supere las $35 \mathrm{kcal} / \mathrm{kg} /$ diarias; el consumo de proteínas debe ser llevado a sus niveles normales $1.2-1.7 \mathrm{~g} / \mathrm{kg}$ de peso), pues estos pacientes suelen ingerir cantidades superiores, siendo frecuente el consumo de batidos proteicos como base de su alimentación, lo cual conlleva a un aumento en los niveles de este nutrimento alterando el metabolismo del cuerpo. En cuanto a los hidratos de carbono estos no deben superar el $50-55 \%$, prefiriéndose aquellos como el arroz, la pasta o papa. Las grasas (20$25 \%$ ) deben provenir principalmente de aceites como lo es el de oliva o de frutos secos, y aumentar el consumo de ácidos grasos monoinsaturados. Además, el nutriólogo debe proporcionar información y formación sobre la alimentación en deportistas para llevar una correcta implementación de la misma ${ }^{46}$.

\section{Conclusión}

En la actualidad los hombres en su mayoría jóvenes, han modificado sus estilos de vida, sobre todo en cuanto a su dieta y ejercicio con la finalidad de mejorar su composición corporal y en consecuencia su imagen, sin embargo, una parte de esta población se ve implicada en el desarrollo del trastorno de Dismorfia Muscular (conocida coloquialmente como vigorexia). Estos individuos suelen caracterizarse por un autoconcepto corporal insatisfactorio, a consecuencia de una baja valoración física, ya que presentan una distorsión corporal percibiéndose a sí mismos con poca musculatura.

La etiología de la DM es el resultado de una conjunción de factores de riesgo de naturaleza biológica, conductual, cognoscitiva, afectiva y sociocultural.

Con los avances tecnológicos, los medios de comunicación tienen un mayor alcance y juegan un papel muy importante en relación a la imagen corporal, ya que imponen estereotipos poco realistas e inalcanzables lo cual ha favorecido la aparición de esta patología.

Estos pacientes suelen utilizar sustancias ergogénicas de manera no controlada e incluso sin una supervisión profesional, con el fin de obtener una mayor masa muscular con resultados más rápidos, sin saber o negando el riesgo de lo que esto puede ocasionar en su salud y bienestar.

Pocos son los programas de prevención de DM un ejemplo de ellos es The Body Project: More Than Muscles, intervención basada en disonancia cognitiva para reducir el impacto de los factores de riesgo asociado a dismorfia muscular en hombres con insatisfacción corporal. El PECANSS es un programa mexicano que incluye estrategias psicoeducativas para la prevención de 
conductas alimentarias de riesgo y obsesión por la musculatura. Sin embargo, es necesario la implementación de más programas sobre todo enfocados a la prevención de dismorfia muscular, pues es muy escasa la evidencia científica que existe actualmente.

En cuanto al tratamiento, se requiere de un equipo multidisciplinario altamente capacitado y ético, lo que resulta ser muy costoso puesto que no solamente hay que modificar los patrones alimentarios y de pensamiento obsesivo, sino que también la mayoría de las veces se realiza la preinscripción de diversos medicamentos.

\section{Referencias}

[1] López C., Vázquez R., Mancilla JM. Evaluación diagnóstica de la Dismorfia Muscular: una revisión sistemática. Anales de Psicología. 2016; 32 (2): 405-416.

[2] González I., Fernández JG., Contreras OR. Contribución para el criterio diagnóstico de la Dismorfia Muscular (Vigorexia). Revista Psicología del Deporte. 2012; 21 (2): 351-358.

[3] Baile J. Dismorfia muscular: definición y evaluación. Psychologia: Avances de la Disciplina. 2011; 5 (1): 135-136.

[4] Mazón J., Atiencia L., Cabrera H. Factores psicológicos asociados a la vigorexia en los usuarios de gimnasios del Azuay en el periodo 20162017. Revista Electrónica de Psicología Iztacala. 2017; 20 (3): 130144.

[5] American Psychiatric Association. Diagnostic and Statistical Manual of Mental Disorders. 5th ed. Washington DC; 2013.

[6] Murray S., Rieger E., Youyz S., De la Garza Y. Muscle Dysmorphia and the DSM-V Conundrum: Where Does It Belong? A Review Paper. International Journal of Eating Disorders. 2010; 43 (6): 483-491.

[7] Vaquero-Cristóbal R, Alacid F, Muyor J, López-Miñarro P. Imagen corporal; revisión bibliográfica. Nutrición Hospitalaria. 2013; 28 (1): 27-35.

[8] Martínez E, Fernández B, Cadena S. Revisión sistemática de la bibliografía del periodo 2006-2016 sobre la dismorfia muscular: prevalencia, herramientas diagnósticas y prevención. Nure Investigación. 2017; 14 (90): 1-16

[9] Cortez D., Gallegos M., Jiménez T., Martínez P., Saravia S, Cruzat C, et al. Influencia de factores socioculturales en la imagen corporal desde la perspectiva de mujeres adolescentes. Revista Mexicana de Trastornos Alimentarios. 2016; 7(2): 116-124.

[10] Hernández A. Detección de síntomas de Dismorfia Muscular en atletas [doctorado]. Universidad de Castilla La Mancha; 2017.

[11] Escoto M., Camacho E., Álvarez G., Díaz F., Morales A. Relación entre autoestima y síntomas de dismorfia muscular en varones fisicoconstructivistas. Revista Mexicana de Trastornos Alimentarios. 2012; 3(1):1-5

[12] Santellán M. La cultura de la delgadez. [Consultado 27 Ago 2020]. Disponible http://www.centrod.apcecom.ar/la_cultura_de_la_delgadez.htm.

[13] Universidad de la República Uruguaya. Vigorexia, una mirada desde la terapia cognitivo-conductual [Consultado 27 Ago 2020]. Disponible en:

https://sifp.psico.edu.uy/sites/default/files/TrabajosfinalesArchivos/tfg _ceresa.pdf

[14] Muñoz E., Gómez P. Adicción al deporte y déficit de atención selectiva. Encuentros en Psicología Social. 2003; 1: 74-76.
[15] Alonso J. La adicción al gimnasio: Vigorexia o Complejo de Adonis. [Consultado 27 Ago 2020]. Disponible en: http//www.mifarmacia.es/contenido/articulos/articulo_ef_vifgorexia.h tm.

[16] Martell C. Vigorexia: enfermedad o adaptación. Revista Digital de Deportes. 2006; 11 (99): 114-119.

[17] Rodríguez J. Vigorexia: adicción, obsesión o dismorfia; un intento de aproximación. Salud y drogas. 2007; 7 (2): 289-308.

[18] Arellano O., Vázquez M., Fernandez T., Saucedo-Molina T. Drive for muscularity and disordered eating behaviors and its relationship with anthropometric indicators and physical activity in Mexican adolescent men. Eating and Weight Disorders- Studies on Anorexia, Bulimia and Obesity. 2019; 24: 661-670.

[19] Camacho E., Escoto M., Contreras G., Ibarra M., García J. Relación entre motivación por la musculatura e indicadores antropométricos en fisicoconstructivistas. Revista Mexicana de Investigación en Psicología 2012; 4 (1): 112-119.

[20] Organización Mundial de la Salud. Estrategia mundial sobre régimen alimentario, actividad física y salud 2004. [Consultado 31 Ago 2020]. Disponible en: https://www.who.int/dietphysicalactivity/pa/es/.

[21] Márquez S., Vega R. La adicción al ejercicio: un trastorno emergente de la conducta. Nutrición Hospitalaria. 2015; 31(6): 2384-2391.

[22] Cantón E., Checa I. El consumo de esteroides y su relación con variables psicológicas en practicantes de musculación. Salud y Drogas. 2011; 11 (2): 129-143

[23] Palacios N., Manonelles P. Ayudas ergogénicas nutricionales para las personas que realizan ejercicio físico. Revista Archivos de Medicina del Deporte. 2012; 39:1-76.

[24] García, J., Álvarez, G., Camacho, J., Amaya, A., Mancilla, J. Dismorfia muscular y uso de sustancias ergogénicas. Una revisión sistemática. Revista Colombiana de Psiquiatría. 2017; 46 (3):168-177.

[25] Blasco R. Las ayudas ergogénicas nutricionales en el ámbito deportivo. Revista Nutrición Clínica en Medicina. 2016; 10 (2): 69-78.

[26] Zurita R. Efectos de ayudas ergogénicas sobre el organismo en relación con el rendimiento deportivo. Revista digital 2009; 19.

[27] Gracia S., Camacho I., Gracia Y., González P., Esquivel P. Consumo de Suplementos Alimenticios en la Población del Área Metropolitana de la Cd. de Monterrey, Nuevo León, México. [Consultado 13 Sep 2020]. Disponible en: http://www.fcq.uanl.mx/wpcontent/uploads/2015/05/32RC-OT_SG.pdf.

[28] Real Academia Española. Anfetamina. Madrid: Diccionario de la lengua española. [Consultado 27 Ago 2020]. Disponible en: https://dle.rae.es/anfetamina.

[29] Martínez A., Cortes E., Martinez N., Rizo M. Factores de riesgos nutricionales para dismorfia muscular en usuarios de sala de musculación. Nutrición Hospitalaria. 2015; 31(4): 1733-1737.

[30] Avella R., Medellín J. Los esteroides anabolizantes androgénicos, riesgos y consecuencias. Revista UDCA. 2012; 15: 47-55

[31] Pope H., Phillips K., Olivardia R. The Adonis Complex: The Secret Crisis of Male Body Obsession Looking Good: Male Body Image in Modern America Making the Body Beautiful: A Cultural History of Aesthetic Surgery Body Modification. Psychiatric Services Journal. 2000; 54 (2): 255-256.

[32] Pope H. Adonis Complex: How to Identify, Treat and Prevent Body Obsession in Men and Boys 2002. Boston: Touchstone Books.

[33] Olivardia R. Mirror, mirror on the wall, who's the largest of them all? The features and phenomenology of muscle dysmorphia. Harvard Journal of Psychiatry 2001; 9: 254-294

[34] Baile J. Vigorexia: Cómo reconocerla y evitarla. Madrid: Síntesis; 2005. 
[35] García M., Toledo F. Nuevas adicciones: Anorexia, bulimia y vigorexia. [Consultado 17 Sep 2020]. Disponible en: http://eoepsabi.educa.aragon.es/descargas/H_Recursos/h_6_Psicol_Cl inica/h.6.1.Materiales_divulgacion/4.11.Anorexia_bulimia_vigorexia. pdf.

[36] Méndez R. Dopaje en el gimnasio. [Consultado 17 Sep 2020]. Disponible

en https://elpais.com/diario/2002/09/16/sociedad/1032127205_850215.ht $\mathrm{ml}$.

[37] Orrit G. Dismorfia Muscular: Factores De Riesgo Y Protectores En Adolescentes [doctorado]. Universidad Católica de Valencia; 2019.

[38] Guerola E., Pejenautle M. Vigorexia. Formación Médica Continuada en Atención Primaria. 2018; 25 (5): 262-9.

[39] Brown TA., Forney KJ., Pinner D., Keel PK. A randomized controlled trial of The Body Project: More Than Muscles for men with body dissatisfaction. The International journal of eating disorders. 2017; 50(8): 873-883.

[40] Saucedo-Molina TJ., Villareal M., Oliva LA., Unikel C., Guzmán RME. Disordered eating behaviours and sedentary lifestyle prevention among young Mexicans: a pilot study. Health Education Journal. 2018; 77(8): 872-883

[41] Salaberría K., Borda M., Amor P., Echeburúa E. Tratamiento del Trastorno Dismórfico Corporal: una revisión crítica. Revista de Psicopatología y Psicología Clínica. 2000; 5 (1): 27-43.

[42] Driesch G., Burgmer M., Heuft G. Body dysmorphic disorder Epidemiology, clinical symptoms, classification and differential treatment indications: an overview. Nervenarzt. 2004; 75: 917-29.

[43] Phillips K. Body dysmorphic disorder. Handbook on ObsessiveCompulsive and Related Disorders. Arlington: American Psychiatric Publishing; 2015.

[44] Synder P. Use of androgens and other hormones by athletes. [Consultado 01 Sep. 2020]. Disponible en: http://www.uptodate.com.

[45] Gómez S., Nova E., Veses A., Gheorghe A., Marcos A. Nutrición y trastornos del comportamiento alimentario. Manual Práctico de Nutrición y Salud. [Consultado 15 Sep 2020]. Disponible en https://digital.csic.es/bitstream/10261/54320/3/Nutricion_y_trastorno_ del_comportamiento...pdf.

[46] Morcillo M. Nutrición y Trastornos de la Conducta Alimentaria [Consultado 15 Sep 2020]. Disponible en http://dehesa.unex.es/bitstream/handle/10662/10112/TFGUEX_2019_ Morcillo_Chamizo.pdf?sequence=1\&isAllowed=y . 